INPLASY

PROTOCOL

To cite: Yang et al. Prognostic value of preoperative assessment of left ventricular function in patients undergoing percutaneous coronary intervention. Inplasy protocol 202220031. doi:

10.37766/inplasy2022.2.0031

Received: 11 February 2022

Published: 11 February 2022

Corresponding author:

Zhi Cheng Jing

jingzhicheng@vip.163.com

Author Affiliation:

Peking Union Medical College

Hospital.

Support: None.

Review Stage at time of this submission: Data analysis.

Conflicts of interest:

None declared.

\section{Prognostic value of preoperative assessment of left ventricular function in patients undergoing percutaneous coronary intervention}

Yang, $\mathrm{M}^{1}$; Guo, $\mathrm{F}^{2}$; Jing, $\mathrm{Z}^{3}$.

Review question / Objective: This study aims to explore the significance of left ventricular function assessment before $\mathrm{PCI}$ by comparing the differences in short - and long-term PCI outcomes between patients with different left ventricular ejection fraction stratified preoperatively.

Condition being studied: Preoperative assessment of left ventricular function in patients undergoing percutaneous coronary intervention.

Information sources: PubMed and Scopus were searched to identify potential studies from January 1, 2001 through January 1, 2022. There were no language restrictions. The reference list of previous systematic reviews were scrutinized.

INPLASY registration number: This protocol was registered with the International Platform of Registered Systematic Review and Meta-Analysis Protocols (INPLASY) on 11 February 2022 and was last updated on 11 February 2022 (registration number INPLASY202220031).

\section{INTRODUCTION}

Review question / Objective: This study aims to explore the significance of left ventricular function assessment before $\mathrm{PCI}$ by comparing the differences in short - and long-term $\mathrm{PCl}$ outcomes between patients with different left ventricular ejection fraction stratified preoperatively.
Rationale: Recent studies have shown that increased use of percutaneous coronary intervention $(\mathrm{PCl})$ reperfusion has led to a decrease in acute coronary syndrome mortality. In fact, patients may experience a decline in cardiac function even after successful primary PCI. Unfortunately, the assessment of left ventricular (LV) function before $\mathrm{PCl}$ is often overlooked. Recent 
studies have shown that increased use of percutaneous coronary intervention reperfusion has led to a decrease in acute coronary syndrome mortality. In fact, patients may experience a decline in cardiac function even after successful primary PCI. Unfortunately, the assessment of LV function before $\mathrm{PCI}$ is often overlooked.

Condition being studied: preoperative assessment of left ventricular function in patients undergoing percutaneous coronary intervention.

\section{METHODS}

Participant or population: Patients undergoing percutaneous coronary intervention.

Intervention: Patients with different left ventricular ejection fractionstratified.

Comparator: Patients with different left ventricular ejection fractionstratified.

Study designs to be included: Observational studies or secondary analysis of intervention studies that reported prognosis of $\mathrm{PCl}$ surgery were included.

Eligibility criteria: Observational studies or secondary analysis of intervention studies that reported prognosis of $\mathrm{PCl}$. Outcomes of studies must be stratified according to LVEF.

Information sources: PubMed and Scopus were searched to identify potential studies from January 1, 2001 through January 1, 2022. There were no language restrictions. The reference list of previous systematic reviews were scrutinized.

Main outcome(s): The primary outcome was all-cause mortality stratified according to LVEF at baseline.

Additional outcome(s): The secondary outcomes were MACE and cardiac mortality in-hospital or long-term.
Data management: Two investigators performed title / abstract screening independently from each other. After that, the full-text of potentially eligible studies was accessed by two investigators for finally determining eligibility and, then, proceeding data extraction. Extracted data included study design, age, gender, grouping rules, sample size, patients, country, follow-up periods, and study results. If the article did not provide data results, we used free software Engaugedigitizer (https://github.com/ markummitchell/engauge-digitizer/tree/ v12.2.1) to obtain data from figures.

Quality assessment / Risk of bias analysis: Study quality was assessed using items from the Newcastle-Ottawa Quality Assessment Scale(NOS). Publication bias was assessed using the Begg rank correlation test and the Egger weighted linear regression test for implementation strategies with at least 10 studies.

Strategy of data synthesis: Random-effects or fix-effects meta-analysis was conducted of outcomes for which at least 2 studies contributed data. Categorical data were expressed as the pooled odds ratio (OR) or Hazard ratio (HR) with their $95 \%$ Cls using the inverse variance method. Heterogeneity was evaluated using both the $X^{2}$ test and the 12 statistic. All statistical tests were two sided and used a significance level of $P<$ 0.05. We used STATA 15 (StataCorp, College Station, TX) for all statistical analyses.

Subgroup analysis: Three subgroups were analyzed: 1. Patients with heart failure (New York Heart Association or Killip class $>1$ ) at baseline, heart failure with reduced ejection fraction (HFrEF) versus heart failure with preserved ejection fraction (HFpEF); 2. Patients undergoing elective $\mathrm{PCl}$ of chronic total occlusion (CTO); 3. STEMI patients.

Sensitivity analysis: None.

Language: No language restriction.

Country(ies) involved: China. 
Other relevant information: None.

Keywords: percutaneous coronary intervention; left ventricular ejection fraction; prognostic.

Contributions of each author:

Author 1 - Ming Yang - The author drafted the manuscript.

Email: yangming@pumch.cn

Author 2 - Fan Guo - The author provided statistical expertise.

Email: guofan1943@qq.com

Author 3 - Zhi Cheng Jing - The author contributed to the development of the selection criteria, and the risk of bias assessment strategy.

Email: jingzhicheng@vip.163.com 